\title{
Beyond Trial \& Error: Iteration-to-Learn using Computational Paper Crafts in a STEAM Camp for Girls
}

\section{Colin Dixon, Concord Consortium}

Colin Dixon holds a Ph.D. in Learning \& Mind Sciences from the University of California, Davis. He researches the development of STEM practices and agency among young people creating things to use and share with the world. He writes about equity and identity in making and engineering, the role of community in science learning, and how youth leverage interests and experiences within STEM education.

\section{Dr. Corey T. Schimpf, The Concord Consoritum}

Corey Schimpf is a Learning Analytics Scientist with interest in design research, learning analytics, research methods and under-representation in engineering, A major strand of his work focuses on developing and analyzing learning analytics that model students' cognitive states or strategies through fine-grained computer-logged data from open-ended technology-centered science and engineering projects. His dissertation research explored the use of Minecraft to teach early engineering college students about the design process.

\section{Ms. Sherry Hsi, Concord Consortium}

Dr. Sherry Hsi is the Executive Vice President of the Concord Consortium. She leads the strategic development, design, and research of learning technologies using her background in engineering, science education, and the learning sciences to improve learning and engagement in STEM. Her work incorporates sensor technologies, computationally-enhanced papercraft, and augmented reality among other technologies. She is the Co-PI of Paper Mechatronics project and the PI of an NSF STEM+C project on computational thinking in high school biology. 


\section{Beyond Trial \& Error: Iteration-to-learn using computational papercrafts in a STEAM camp for girls}

\section{Introduction}

In professional engineering design process, iteration is critical to improving design outcomes and takes different forms at different points during idea generation, prototyping, and refining stages. In engineering education, iteration is a desired and encouraged step for learning valuable design practices that include testing, troubleshooting, and problem-solving under uncertainty and multiple design and resource constraints. In the face of novel and multifaceted problems, future engineers must be comfortable and competent not just trying again, but taking initiative to identify failure points, adjust goals, troubleshoot effectively, and run useful tests. Yet, when asked about how they deal with unforeseen problems, novice designers often describe a process of "trial and error." This process might fairly be described as iteration, but falls short of the practices that engineering education needs to develop. This paper shares an exploratory study of elementary school girls who engaged in a design activity using computational papercrafts in the context of a "STEAM" summer camp. Drawing from qualitative analysis of student interviews, classroom observations, and student-designed artifacts, we aim to better understand and support iteration for learning in computational papercrafting. We show where participants engaged in testing and refinement and how this benefitted the participants' projects and their learning. The resources and practices described here are especially relevant within making-oriented engineering education and other settings working to broaden participation in engineering.

\section{Iteration in engineering education}

Iteration is central to engineering design and enables rapid cycles of improvement towards innovative solutions. However, iteration in engineering is described at different grain sizes of the design process and subsequently has many definitions within the fields of education and design, as well as within research [1], [2]. Where some see iteration as inextricably linked to action [3], others see it as primarily conceptual and more open-ended, a chance to explore all possible designs for a given problem [2]. Iteration can focus on problem framing, or on refinement of the solution [1], [4]. It can happen around small problems within stages of the design process as designers troubleshoot, tweak, and make incremental improvements, or across stages in ways that lead to large changes or redefine projects entirely [1], [4], [5]. Iterations may happen on a component of a design artifact, on several components of a design artifact or could result in a new cycle through the entire design process to create a new artifact that relates to a prior design.

Iteration is also a sociotechnical process with a set of practices where a design practice or set of design practices are repeatedly applied to some part of a design artifact [1], [3], [6]. This process can be viewed as the repetition of particular design practices or as repeated operations on a design artifact [1], [4], including prototypes, mock-ups, and sketches or other representations.

In the context of designing quality STEM learning experiences (that can have consequential outcomes for all students), we consider iteration as processes through which learners rework, rethink or revise their ideas during a student-centered hands-on design project. Iteration can be done individually, or in collaboration with peers or mentors, with elements (like critique, 
ideation) distributed across tools and teams. To support the iteration of their projects, they may engage in new practices - like testing, prototyping, and research - or generate new prototypes or representations.

Sophisticated processes of iteration envelope one or several other practices, such as testing, discussion, help-seeking, research [7], [8] and may include some of the practices described in the NGSS Framework. For example, the NGSS Framework, 3-5-ETS-3, states that a learner needs to "plan and carry out fair tests," which involves identifying failure points and difficulties, then identifying and gathering relevant data [9]. Designers are intentional and can explain the design rationale with each suggested iteration. Experienced designers run quick, valid tests, conduct focused diagnostic troubleshooting, and actively look for potential faults. These help them move efficiently through progressively better ideas and prototypes. This stands in contrast to naive designers who tend to run random and confounded experiments, brush aside unanticipated results, and often remain intent on original ideas [8], [10]. Though novice engineers may be engaging in iteration, they may not be able to explain their choices and their practices included in those iterations may be less sophisticated.

Iteration in design projects may happen for several reasons. A common reason is that the problem is not fully understood, leaving room for the designer to come to a deeper understanding of the problem while designing [2]. With a better understanding of the problem or the design space it may be necessary to iterate over aspects of the design. Similarly, iteration can happen when new information is discovered, pushing the designer to improve designs [1] or generate new designs [4]. Also common are corrective iterations that happen when a problem with their design or plan appears and stalls progress [1], [11].

\section{The limitations of iteration}

While iteration can benefit the design process and product that emerges from it, iterations can also increase the cost and time to finish a design project [1], [5], [6], [11] and do not always improve final design quality [4], [6], [11]. This is especially true for novices who may rush into "trial-and-error techniques" with material construction and reconstruction without sufficient time dedicated to evaluating problems and potential solutions [8], [12], or who may rely on testingand retesting when problems become complex, instead of gaining appropriate conceptual knowledge [13]. When iteration is made necessary by an oversight or not being careful in one's design, it may exhaust students' time for a project in ways that are avoidable and do not move their knowledge or the project forward. Unproductive practices are easy to replicate and difficult to explicitly address in engineering education classrooms when teachers are time- and resourceconstrained.

Wynn and Eckert [1] describe a particular type of corrective iteration they call churn, where parts of a problem are revisited repeatedly without landing on an adequate refinement. Novice designers can also fixate on certain parts of the design, such as information gathering, modeling, or particular features of an object [14]-[16]. In such cases, designers must be able to switch focus or strategies in order to move forward.

The presence and duration of iterations can be influenced by attributes of the design situation [6] and that interventions can affect iterations happening in a design situation [1]. A large body of 
work focuses on minimizing the negative aspects of iterations in professional design settings through various prescriptive models [5], [11], [17], while a smaller subset of work has emphasized the positive attributes of iteration in professional settings [18]. While some of the studies discussed above provide some recommendations for how to support iteration, there is still work to be done to clarify the goals and practices for iteration should be within the context of engineering education. How does, and should, iteration look like for novice designers? What practices are important to introduce and support? When is iteration effective, for learning and for developing a design idea or artifact?

\section{Iteration-to-learn}

The discussion to this point, coming largely out of analyses of professional practice and university design courses, reflects what we are calling product-oriented iteration. The primary value of iteration is to improve designs and artifacts or solve problems, and in service of those goals, to build understandings of materials or tools. In this view, while iteration is central to engineering design, it should be employed only when efficient and effective, and the drafts left behind are erased in presentation of the final artefact.

In contrast to product-oriented iteration in which iteration is complete or successful only when it leads to decisions or improvements [3], in person-oriented iteration, or iteration-to-learn, the chance to redo and revise provides novice designers with an opportunity to (a) be introduced to practices and tools they may not otherwise have encountered, and (b) develop interests, social positions, and connections that can support longer-term participation in engineering and related domains. These goals do not preclude improvement of the product or work toward solution of a problem, but are prioritized when creating structures and supports for design and engineering work.

Because young people coming into maker or engineering spaces may be unfamiliar with tools and may not initially understand affordances of these spaces, we see iteration as an important chance for learners to build and exert agency. Learners can use iteration to come back to project plans and problems with a clearer sense of what opportunities they present, and to use this understanding to reshape the path of a project to align with personal goals and existing areas of expertise. In this view, iteration also gives novices a chance to enrich conceptual knowledge around topics or tools of interest, or to pursue social positions and relationships, such as the chance to work with a particular friend or mentor, or chance to get recognized as an expert in some area. Designers can expand or narrow vision of a project, and redirect toward new resources - whether human or material.

Drawing from research about the value of "tinkering" [19], this perspective emphasizes an "iterative approach" in which iteration is not a just a step in product development, but is woven throughout the practice and culture of the learning environment. Facilitators use iteration work to open up more diverse, more inclusive learning pathways, to support persistence, and to facilitate development of practices and skills [20], [21]. Mistakes are celebrated and previous drafts and designs are displayed with sense of accomplishment [22].

With an iteration-to-learn orientation, specific practices of iteration are not assigned or required, but rather emerge as learners pursue project goals. If required as a prerequisite to building or as 
compulsory steps regardless of relevance to student goals and project, practices central to engineering may be perceived as arbitrary or a waste of time. Requiring students to engage in steps that delay direct engagement with materials, may also limit the value of maker-oriented education to expand participation in engineering.

However, iteration can (and should) involve valued practices, like systematic testing of abstract models, generating and using data, and verifying known outcomes [7]-[9], [31], that learners may not have engaged with in their initial design work. While we caution against overspecifying steps of iteration, under-specifying our goals for iteration may mean that we fail to sufficiently support student trajectories inside and beyond engineering and design. Failing to identify and name valued practices can also mean losing a link between accomplishments within making-oriented learning and positive learning outcomes across STEM settings [22].

If iteration is under-supported, learners may engage in iteration without noticing key problems and affordances, and without avoiding fixation or deepening knowledge of materials and tools [30], as can be the case with "churn" and trial-and-error iteration. The use of the term "trial-anderror" to refer to iteration within making and tinkering contexts remains common, particularly informally among educators (e.g. [23], [24]), and youth (e.g. [25], [26]). Some educators point to "test and check" - just trying something to "see what happens" - as a useful way to gain familiarity with tools or techniques (e.g.[27]), and debug some kinds of technical or procedural problems [28], especially when interspersed by expert support. If we do not articulate and design for iteration beyond trial-and-error, we hinder development of systematic troubleshooting and revision practices, and jeopardize connections to practices of engineering and other STEM fields that iteration might otherwise open [25], [29]. Instead of prescribing practices of iteration, educators can support them with pedagogical resources and structures, such as displaying drafts, encouraging cultures that value and build on mistakes, providing testing stations and troubleshooting routines [8], and lengthening "horizons of observation" that allow novices to see how experts test and refine [38].

Each iteration represents an opportunity to deepen participation in engineering endeavors - a chance to learn new ways of seeing and to align powerful disciplinary practices and tools with goals that young people bring to and build within making, tinkering, and engineering. Iteration gives the educator a chance to motivate practices as response to real needs and project goals that are visible to the learner. Fostering this has implications for K-12 engineering education that not only supports development of engineering practices but also fosters broader and longer-term participation in engineering and related disciplines.

In light of the reality that iteration can be both a positive and negative force within a design situation and the limited research, outside of professional settings, about what aspects of a design situation may affect positive and negative iteration cycles, in this exploratory work we ask:

1) What opportunities for testing and refinement did computational papercrafts open up?

2) What resources and tools do young learners employ when testing and refining designs?

We focus on testing and refinement as crucial parts of successful engineering design, particularly in complex projects and when engaging with systems or materials that are unfamiliar - the type of novel problems with which we want future engineers to be comfortable and competent. From 
the standpoint of study methods, we focus on the study of testing as one aspect of iteration because the intention and actions taken by learners are observable by researchers. The reasoning behind students' choices of testing approaches and tool selection provide insight into student goals for intended refinement.

By exploring these questions, two important insights may be gained. First, what aspects or components of the learning environment, and by extension design situation, encourage or compel student iterations? And second, what do student-led iterations look like? Insight in regards to the first point may help refine scaffolding of iteration for student learning. In regards to the second point, insight into the nature of student iterations will deepen our understanding of how students navigate complex challenges, including how they balance social, technical, and epistemological concerns in a design situation with structured support but high autonomy and many multiple possible paths [39]. This will help educators to design supports that align valued practices of engineering with young people's practices and preferences, instead of supplanting them.

\section{Context \& methods}

\section{Design Conjectures}

For this study, we selected a computational papercraft activity because it lowers cost and intimidation. Everyday materials found in school classroom, libraries, or homes form the basis of building materials such as cardboard, glue, tape, and straws. Combined with hobby electronics, motors, and microcontrollers, papercrafted sculptures or paper machines can be made accessible to children and playful to move, wiggle, or ambulate (see [32]). Our design conjecture was that by making materials familiar and abundant, learners could easily modify and replicate components and would be more likely to engage in testing and refinement. With relevant prior experience, they would also be better able to recognize potential faults in their design and identify areas in which they could build on existing knowledge.

We also supported design with a free and open source, web-based gear simulator called FoldMecha [33] and a customized circuit board, the LocoTap. Using FoldMecha for modeling mechanical motions students we hoped that participants could familiarize themselves with relative motions of gears, cams, and linkages. With the LocoTap, students could quickly plugin and connect servos to test the motorized movements. Microcontrollers were left at multiple testing stations around the room to enable students to engage in testing and troubleshooting at any time during the design process (cf. [8]). We adopted this strategy since forcing iteration particularly when participants perceive the time period as too short - can curtail exploration of multiple project routes [30].

\section{STEAM Camp}

To examine the practices of novice designers when testing and refining ideas and prototypes, we conducted qualitative analysis of structured interviews, video, and designs of four girls, ages 9 11 , out of eleven total participants working on computational papercrafts as part of a museumbased STEAM (science, technology, engineering, arts, and math) summer camp. 
Camp Context: Girls were enrolled in a week-long camp, hosted by a museum. The theme of the camp was "The Fun of Flight" in which campers engaged with different science, arts, and craft projects related to flying machines and aerodynamics. This theme provided a framing and direction for students to begin to imagine and create a paper creature. Following a comparable approach to open-ended design projects in K-12 settings, e.g. [2], the theme scaffolded engagement in the project by narrowing consideration of design directions, but participants could go outside the theme if they had a compelling design idea they wanted to pursue.

We introduced a computational papercraft project that asked girls to make a paper mechatronic bird of their choice. This involved design and construction of paper wings and cardboard sculptures with gears and linkages powered by servo motors. Students first made hand-sketches of creatures they wanted to create, and then used FoldMecha, as well as discussion with peers and mentors, to imagine the type of motion their creature would have. Over the course of one day, the girls generated designs inspired by (but often straying beyond) the project theme, then worked with mechanics, electronics and craft to create working versions that would be displayed as part of a public exhibit. Students continued to tinker with their designs over two additional days during the camp.

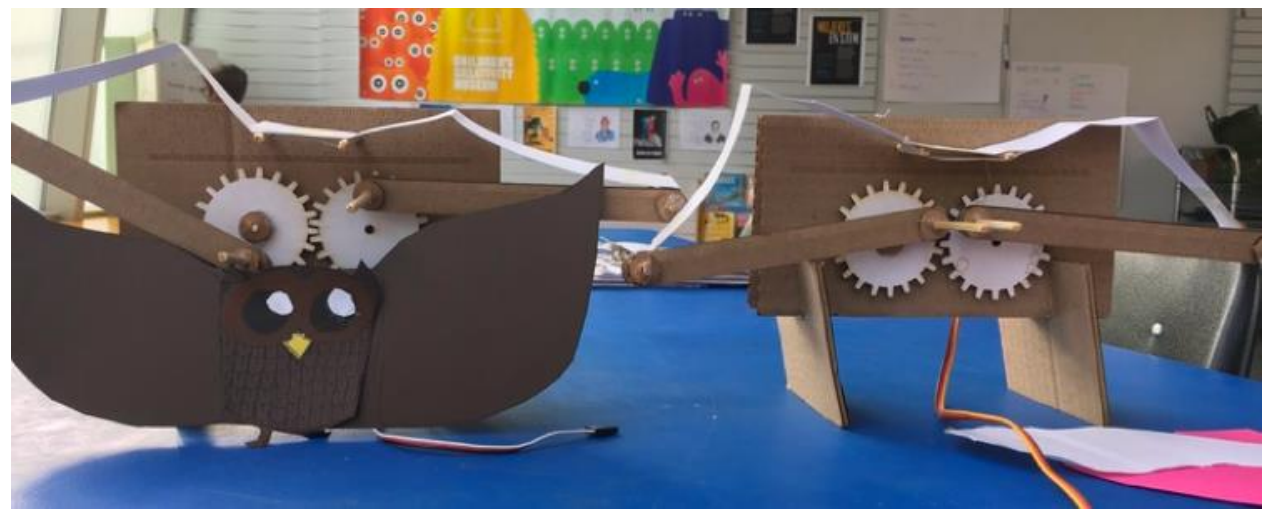

Figure 1: An owl and underlying mechanism - designs made by girls using FoldMecha, LocoTap microcontroller, and paper components.

Demographics: Participants were from the urban area around the museum, and included girls from middle to upper middle-class families. Asian, Caucasian, and East Asian/Indian participants were represented.

Facilitators: The camp was staffed by one main camp instructor and three assistants, who ranged in age from 17 to 25 . All were women. While one assistant had some prior experience with computational papercrafts, other instructors were new to the field and tools, but had experience with education, engineering, and/or crafting. In addition to staff, two researchers (one woman, one man) and one designer (a woman) with more experience in computational papercrafts, and these tools in particular, introduced the workshop and assisted participants for part of the first day of their work. 


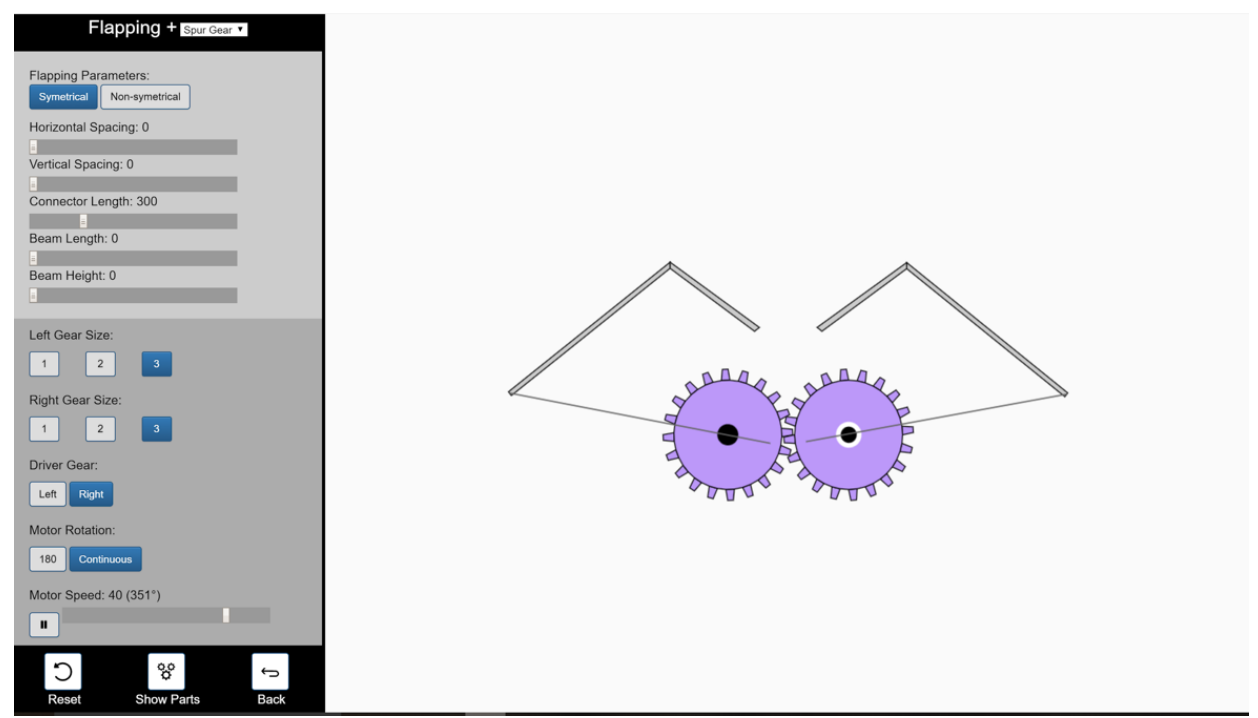

Figure 2: Flapping mechanism simulator

\section{Data Collection \& Analysis}

We collected several forms of data during the workshop, including students' intermediary design documents, students' final artefacts, audio recordings, and video recordings, as well as teacher and student interviews. Two types of data were of primary interest for this study: retrospective interviews and audio recordings. Four students participated in retrospective interviews to share how they designed their birds, what challenges they faced, and what resources they drew on when facing initial design and subsequent refinement. Additionally, we placed audio recorders at each workstation to capture interactions and discussions while working on their designs. Video recording captured some of the girls' work, but due to culture of the workshop, participants moved around the room frequently and a complete video record of work was not possible. Collecting these types of data allowed us to analyze reflections and practices that spanned longer segments of the project, while also documenting moment-by-moment action and interaction.

We conducted data analysis using thematic analysis of workshop participants retrospective interviews and transcribed audio recordings. Thematic analysis [34], [35], is a broad qualitative method used to distill data into a set of common or pronounced categories or themes. First, we analyzed retrospective interviews inductively to identify practices and ways of talking about iteration common across participants. This resulted in a set of preliminary themes that we detailed and refined through constant comparative method [37] as we next moved to analyze participant discourse transcribed from the audio logs. We present and describe final themes and examples below.

\section{Findings: Iteration within computational papercraft}

We report on three prominent aspects of iteration practices within computational papercrafting, presenting examples from focal cases along with associated features of the activity and facilitation. 


\section{Focal cases overview}

To illustrate the themes identified, we provide examples from two workshop participants: Carmen and Angelica ${ }^{1}$. Though Carmen and Angelica worked with peers and mentors throughout their projects, we focus on their individual experiences because each played a lead role in developing a computational papercraft project and for each we had interview data that provided insight and grounded our interpretations of observational data.

Carmen worked with two other girls, Phoebe and Shruti, on a team to design a penguin. As the girls explored design possibilities and merged ideas, they wanted to create a penguin whose feet would move, along with the wings, in a wobbling motion that evoked how penguins walk. An example sketch of their penguin is provided in the figure below. With two linkages connected to each gear, instead of just one - as in project examples - the design added complexity to their machine. The group spent a considerable amount of time testing out different means to create this motion.
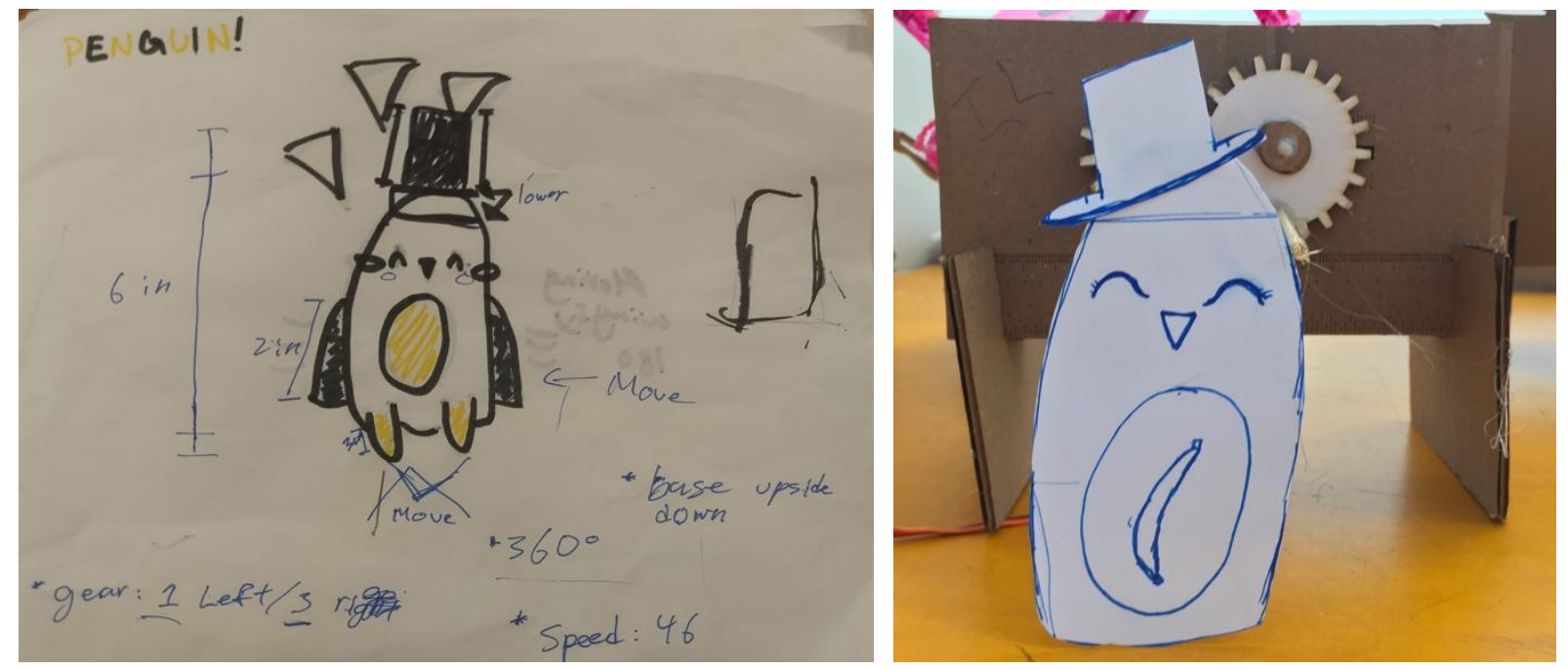

Figure $3 a$ and $3 b$ : Sketch of wobbly penguin and work-in-progress

Angelica made a seal who sat on a bicycle. The primary motion Angelia hoped to represent was pedaling and/or movement of the bike wheels. Unlike other workshop participants who used a motor to power their papercraft, Angelica pursued a hand-crank mechanism for moving the bicycle wheels. In addition to developing and testing the motion of the seal on a bicycle, Angelica spent a large amount of her time designing the shape, size, and appearance of the seal in relation to the bicycle.

\footnotetext{
${ }^{1}$ Pseudonyms ae used for all youth participants and research site locations
} 

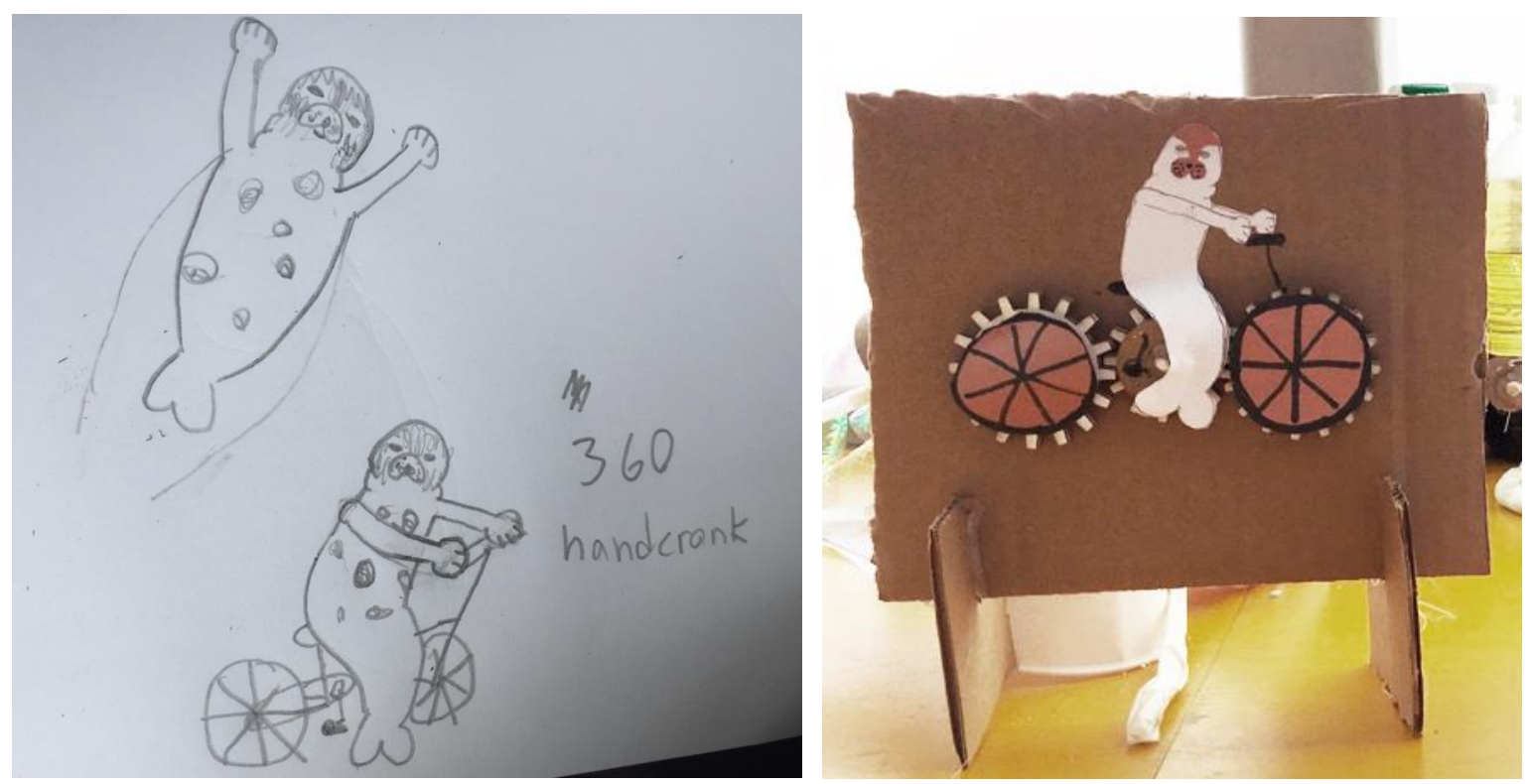

Figure $4 a$ and $4 b$ : Sketch of seal on a bicycle and completed project

Table 1: Summary of iteration-for-learning and focal cases

\begin{tabular}{|c|c|c|}
\hline $\begin{array}{l}\text { Iteration-to-learn } \\
\text { components }\end{array}$ & $\begin{array}{l}\text { Carmen } \\
\text { Project: a wobbling penguin }\end{array}$ & $\begin{array}{l}\text { Angelica } \\
\text { Project: a seal riding a bike }\end{array}$ \\
\hline $\begin{array}{l}\text { Subsystems allow for } \\
\text { stepwise progression }\end{array}$ & $\begin{array}{l}\text { - Tinkering with bird legs and arms } \\
\text { - Refining gear assembly to match intended } \\
\text { motion } \\
\text { - Using a microcontroller to test } \\
\text { mechanism functions }\end{array}$ & $\begin{array}{l}\text { - Using paper gears to understand the } \\
\text { difference in motion between meshed } \\
\text { two gears versus train with three gears } \\
\text { - Making a hand crank to test } \\
\text { - Removing hand crank then testing with } \\
\text { microcontroller }\end{array}$ \\
\hline $\begin{array}{l}\text { Rapid and imaginative } \\
\text { iteration with flexible, } \\
\text { low-cost, materials }\end{array}$ & $\begin{array}{l}\text { - Stacking paper washers to level out } \\
\text { linkage assembly and gears } \\
\text { - Making and attaching different paper feet, } \\
\text { wings, and legs to test } \\
\text { - Testing upside down orientation for bird } \\
\text { base }\end{array}$ & $\begin{array}{l}\text { - Resizing and re-cutting seal and bike } \\
\text { parts to be proportional } \\
\text { - Drawing and redrawing on paper }\end{array}$ \\
\hline $\begin{array}{l}\text { Both technical and } \\
\text { human resources } \\
\text { support simulation }\end{array}$ & $\begin{array}{l}\text { - Using FoldMecha simulator to } \\
\text { troubleshoot motion in their design } \\
\text { - Asking educators to help move the } \\
\text { cardboard parts to show the gear motion }\end{array}$ & $\begin{array}{l}\text { - Using educators to troubleshoot overall } \\
\text { motion in their design } \\
\text { - Asking for help to make the crank } \\
\text { motion work better }\end{array}$ \\
\hline
\end{tabular}

\section{Subsystems allow for stepwise progression}

Two overlapping affordances of computational papercrafts that became important to all students' testing and iteration were (1) the ability to work on subsystems of the project without compromising or delaying work on others, and (2) the ability to try ideas and small changes quickly and with little risk. Addressing the first of these, students tested different subsystems or 
components of their design, from general checks for functionality to specific tests of desired types of motion.

Being able to iterate on parts of the project independently from the whole was particularly important to the students in their work on gears and control. In interviews, the participants recalled "testing" gears and talked, as in the quote below, about being able to move from figuring out how gears would work to testing the motor and back. Asked about how she knew whether her project would work or not, one participant had this to say:

I tested the gears - there's three sets of gears: large, medium, small. I tested those. And then you can test, definitely the motor, cause there's different ways to change the motor, going fast and slow... I [also] checked - I tested - the engine of how fast and how slow it can go.

In computational papercrafts, parts can be easily decoupled, allowing for stepwise testing in which participants can address structural, mechanical and aesthetic elements independently, before putting them together. This allowed progress to continue - by briefly switching to other elements, or through the work of teammates - when sticking points might otherwise have caused frustration or derailed a project vision. It also encouraged participants to conduct focused tests, which brought about greater insight into structural and mechanical elements, and allowed educators to take a heavier hand in moving a project through particularly tricky elements, without detracting from participants' sense of overall ownership.

\section{Carmen}

For Carmen, use of the customized microcontroller - the LocoTap - was central to her ability to figure out how a servo motor worked and then move through gears and connecting parts. In the interview she said: "we did things step by step, so we tested it, like a lot... We tested the LocoTap and motor just by itself too, so we made sure the motor works, so our entire bird can work." As her emphasis (noted with italics) showed, Carmen saw the overall project as dependent on the motor, but separable in a workflow. With her teammates, Carmen was able to try "messing around" with motor speed and gear sizes.

Educators supported this by asking early on if aspects of the project - like gear and "wing" movement - were working like the participants expected, and encouraging them to pay attention to particular aspects, such as "choppiness or smoothness" of motion, and speed of rotation. Another move that was important to successful testing and refinement of subsystems was suggesting ways to make tests visible. For example, early in the day, an educator helped one of Carmen's teammates put a servo horn or propeller on the end of the servo motor. Though a simple move, doing so made it possible for Carmen' teammate to see, where she hadn't been able to before, how the motor in isolation related to her longer-term vision: "when we put the motor on - we put a little circle spinny thing [the servo horn], and it sort of showed what it would look like, sort of - like what speed you want it to go at. So we did test it on that."

Alongside these facilitation moves, testing and being able to mess around in this way helped Carmen to notice and envision singular elements as parts of the whole. It also helped Carmen and her team develop an understanding of how to use these tools and materials and create 
something that matched their vision of a waddling penguin, and led to opportunities to notice and discuss, drawing attention of features, interactions and causal explanations. In one example, one educator worked with Carmen while she was trying out gears of different sizes, and asked her why she thought they were moving at different speeds.

Carmen: Well, the bigger one is pushing the small one, so they get more momentum, and more speed, and this one is giving its energy to the smaller one.

Educator: What would happen is the small one was driving and big was following. Carmen: It would have more momentum, it would switch.

While this initial explanation was incorrect, working through possible gear combinations gave Carmen a chance to improve her explanations. She was invited to test her hypothesis, and subsequent conversations brought her attention to teeth number and circumference.

Angelica

While constructing her seal on a bicycle, Angelica tested out different numbers and configurations of gears to create rotation of the wheels. Like Carmen's group, she isolated this element of the design first and worked to try out different sized gears. She first worked with an educator to figure out how to create a handle for the hand-crank, then tested gear combinations using a base she eventually discarded. She eventually created a line of three gears, so that the two wheels could rotate in the same direction, something she had not thought about when first designing a two-gear system.

As Angelica was building, she was able to ask questions of the visiting designer, who helped her to see new ways of looking at and testing her device, including picking it up to look closely at the back and underneath to see how things fit together. After being satisfied with how the gears moved, Angelica told the designer that she would move on to the draw the seal. The designer then reiterated advice to take time with each element, saying, "when you make things, it's better not to make things too fast."

We also saw the importance of engaging in what felt like "play." Beyond any affective advantages this might have, an ability to test in a fluid way allowed Angelica to think about new possibilities and to imagine the movement of her speedy seal while looking at a lonely spinning motor or the coarse movement of gears. In the interviews, another participant captured this in describing her work with the LocoTap microcontroller: "Like, usually, I don't really test stuff, I just $d o$ it... or either ask people, without even trying with it first. And now - when we did this, I definitely tested a lot of things, like the gears and the motor, and played with the LocoTap a lot."

\section{Rapid and imaginative iteration with flexible, low-cost materials}

The second aspect of iteration we saw overlapped with the ability see and work on subsystems of the overall project, but was supported through different means: participants were able to design, modify, and discard, if necessary, components of their projects with relatively low risk because of the low-cost and biodegradability of paper and cardboard. Cardboard gears, arms, and bases allowed students to test an assortment of design options from motor speed, to gear size and symmetry or asymmetry. While this, to an extent, contributed to the deliberative and conceptual work that we described in the previous section, we saw inexpensive nature of materials being 
most important for participants' refinement of the project aesthetics - how they wanted their birds (and seals) to look.

Participants could run low-cost trials of mechanical configurations or papercraft appearance without concerns about this negatively hampering their design later on, breaking pieces they wouldn't be able to use later, or using up valuable supplies. Girls freely created several versions of their paper creatures and tried multiple configurations of the mechanical papercraft, thereby exploring more possibilities.

Educators encouraged this by recommending that when participants were not sure how something might work, or how they wanted something to look, that they "experiment." Though the design process began with sketching, and educators would often recommend to draw out an idea, or use the simulation tool, the participants' lack of experience with gears and servos meant that this often hit a dead end. Educators helped girls get different pieces and try out a rough version of what they thought might work, "moving them how you want [them to]."

Being able to try out ideas (within focused subsystems or components, as described above), allowed participants to build up intuitions in areas they knew little about and to refine their vision to fit what they were learning about the medium. As the session continued, the participants took up the ethos of trying things once ideas had been discussed. They could be heard suggesting to each other, "let's try it!" when a new solution had been proposed, and asking for teammates or educators to get more supplies.

\section{Carmen}

Carmen's team faced a challenge in trying to create a wobbling motion that involved both wings and feet. Once they had figured out the combination of gears they thought would work, there was still much work to be done: figuring out how to build the penguin around this mechanism, how to create legs and wings that would look and move as expected, and how to attached all the pieces securely, while allowing for movement.

The team was unsure how to build the motion they were looking for, so the low-cost mechanical papercraft gave them opportunities to test and learn which configurations were most promising. As they worked to get the gears and legs moving consistently, they were able to try out small pieces, like cardboard washers, able to glue and take-apart pieces, and able to modify aspects like anchor points, shape, and spacing of the components. Carmen would ask her teammates to get more pieces (and vice versa) as they worked. As the project progressed, they came to look more closely at connections, alignment, how these effected the overall movement and how they could be improved.

However, early on, the inexpensive and malleable cardboard parts also gave them license to think in new ways about the entire configuration. After the teams began constructing basic mechanism, based on the examples, one of Carmen's teammates told an educator, "I want to have the wings and the feet moving." The educator replied, "Ok, that's a good challenge," and began talking through options. However, the teammate stopped, and said, "wait, what if we turn the whole assembly upside down?" Because the bases were simply corrugated cardboard, the 
team could set aside their already begun mechanism and try this new idea, which proved to be successful.

Angelica

Across many iterations, Angelica tried out many different variations on her seal and bicycle, changing the face, head and neck of the seal, as well as its posture and relative size compared to the bike. For Angelica, like Carmen's group, being able to take things apart, examine, and modify was important to refining the seal, and she spent some of her time trying different configurations of the mechanical papercraft, as demonstrated in the interaction below:

Educator: (pointing to part of the mechanism) let me take the top of this to see how it works. It works very well. and when we push it out it works well - the problem, it doesn't match this part."

Angelica: I think we might need to cut some of this off - it's blocking.

However, a considerable part of Angelica's work focused also on the appearance of her seal and bicycle. Again, the low-cost of the papercraft materials gave Angelica opportunities to test and iteratively refine the appearance of her creature. During work, Angelica commented to an educator, "[the seal] is too small. The wheels are ginormous. I'll just make it again. It's going to be giant - well, it's going to pretty big, but not giant." In the interview, she brought this up when talking about what she tested. We saw that through being able to work through designs, Angelica, on her own, came to realize the value of a canonical design and iteration practice: measuring.

Angelica: also, I tested my seal, if it would be big enough. And, so - the first time I made the seal, I put it next to the bicycle, and it was like a perfect seal, and it was way too small.

Interviewer: so, what did you learn from all those tests that you did - like the seal size and the gears and all that?

Angelica: I learned that - I learned that you should always measure stuff before you start. Interviewer: Measure everything?

Angelica: Yeah - pretty much. And also sketch before you start.

Later in the day, when showing the project to another educator, Angelica brought out the first seal, saying, "this is my original seal that was way too tiny.... this one will just be a souvenir."

\section{Technical and human resources for simulation}

In working on their mechatronic creatures, students called upon a variety of resources to advance their design. As we analyzed the reflections and activity of the participants we looked for ways they made use of both material (including technological) and human resources. As one would expect, the wide variety of resources available during the workshop were put to use by the participants in a variety of ways as they worked to envision project ideas that were feasible, troubleshoot spacing and alignment of their gears, connect the servos, and finalize their projects as time ran out. One significant area in which the resources played a role in aiding design and iteration, especially among the relatively novice group, was in simulation of mechanical movements. 
Though the participants were able to draw on prior experience with papercraft as they built their projects, few in the group had experience with gears, linkages, and servo motors. As a result, it was difficult for them to envision how their birds might work and specify designs in ways that would inform subsequent construction. As they iterated both their sketches as well as the projects themselves, being able to create hypothetical models of how mechanism would function was made possible in two main ways: drawing on the knowledge of educators and making use of the FoldMecha online simulation tool.

Though we had originally imagined that FoldMecha would be used just in initial design and planning of the birds, the participants made use of the tool primarily in service of iteration. Rather than inform their first blueprints, the simulator was used to troubleshoot and revise designs only after initial designs were on paper and components had been brought out. As described above, participants sketched ideas, then were invited to make use of the FoldMecha application as they added to their sketches and planned out how the machine would move. However, many were initially unsure of how to best make use of this resources and only briefly engaged with it. When they started to connect gears and create moving parts, many girls returned to the simulator. In response to problems that arose, the participants used the tool to look at how the gears and linkages interacted, and changed parameters (like gears size and symmetry, linkage and wing lengths) to "test if and see if it looked right."

When helping the participants who were note sure how to revise their designs, the educators would also use body movement, refer to examples and analogies and move around gears. They posed "what if" questions and asked the girls to "imagine" what would happen as the gears and linkages interacted. In many cases, the simulation and use of educators supported iteration at the stages of sketching, planning and troubleshooting, minimized frustration and use of time later on.

\section{Carmen}

Early in their design process Carmen's team received advice from a number of educators, who offered questions and recommendations as the team thought through how to set-up and modify their gears and connecting arms. Recommendations included that Carmen look back at FoldMecha. As the group showed two educators how they wanted the penguin to move and where they were getting stuck, one of the lead facilitators, told them, "go to FoldMecha - play with all the variables, all the options - symmetrical, asymmetrical." He showed them where in the application interface to adjust the parameters, helping them see the barebones simulation as the "right wing, left wing." He also encouraged them, once they had a sense of what they wanted, to draw their penguin body directly on top of a printout of the FoldMecha mechanism, "to see what's going to be connected to the beams and what's going to be connected to the gears." In the interview, Carmen recalled using the simulation at this early stage to revise their plan before building, as well as a little later, once they had begun "to make sure it [the penguin] would be wobbling."

As the group moved farther along and begun adding the wings and legs of the penguin, the online simulation became less useful, especially since it did not have the ability to add a second set of linkages to the gears. At this later stage, the team relied more on knowledgeable educators to help them imagine how the final product might look and work. This passage captures another of the educators moving components manually as she described how the mechanism might 
move: "see how the feet are going to come way up? 'see the feet, now you can't.' So as the wings go up, the feet go in. Is that OK with you guys?" In addition to addressing the technical issues, she helps the team see how the technical possibilities might fit into the narrative of their wobbly penguin, suggesting that the motion enabled by the current configuration is "like the feet playing hide and seek."

Angelica

Because she decided to create a hand-crank mechanism and ended up creating a gear train, which FoldMecha did not simulate, Angelica relied primarily on support from educators and peers. Using descriptions as well as mock-ups of the gears, laid out on the table, educators helped her visualize how multiple gears might work and how the seals itself might move.

In both Carmen's case, in which FoldMecha was a central resource, and Angelica's case, in which educators had to simulate mechanism with language or manipulating materials, the participants (including educators) moving frequently back and forth between the simulation and the actual parts (or sketches) that were being developed. This ability to shift easily between what was being imagined and what was being built seemed to be central to productive iteration, supporting both direct improvement of the artefact and more focused analysis and learning on the part of the girls.

\section{Discussion}

Analysis of students' retrospective interviews and audio recording of their design sessions revealed three ways in which testing and refinement were supported in the learning environment, and benefitted both the participants' projects and their learning. These were common across participants of the workshop and the interactions we describe were not out of the ordinary. Below, we briefly discuss ways in which the resources and practices we have described are especially relevant within making-oriented engineering education and other settings working to broaden participation in engineering.

\section{Facilitating Trial-before-error}

When designing and building, engineers need not build something in order to test it. For engineers with sufficient experience in a given domain, mental simulation can act as a proxy for prototyping and testing an idea or direction [30]. However, this kind of mental testing of ideas is not possible when a designer has insufficient experience to make reliable predictions.

In the workshop, participants used the online gear and linkage modeler, FoldMecha, to accomplish this simulation. We had designed this support for initial ideation and planning - to expand participants sense of what was possible and help translate their ideas into the medium. In analysis, we found that the simulation was often used much later in the building process than we had intended.

Participants used FoldMecha to think through what might be happening when they ran into problems - when gears were not fitting together as hoped or not creating an intended movement. They used it to generate new ideas, not at the beginning, but later, when limitations to their 
original visions and specifications were realized. They also used it to test hunches about how something might work or look, such as when Carmen used it to test whether different sized gears would create a desired kind of wobble.

However, we saw limitations to the online tool. Because such tools must be bounded, there will always be cases that fall outside of their scope. As we saw above, this is especially true with creative and open-ended project-based work: Carmen's team flipped their mechanism upside down and wanted to use two linkages per gear, and Angelica created a gear train.

When the limitations of FoldMecha to support simulation were reached, participants relied on more knowledgeable facilitators to simulate consequences of proposed design solutions. They did this by creating mock-ups and alternate representations, using gestures, describing verbally what could be expected, and asking what if questions to refine participants' design space. Dynamic representations and mock-ups can function to support simulation and testing of ideas, and help overcome limitations due to cognitive load [30]. Similarly, we believe these social supports are important for overcoming limitations due to lack of experience with particular materials, tools, or interactions, in this case the working of gears and control of servo motors.

Because building and testing a gear-linkage configuration would take time and introduce imprecision that would confound a test, simulations worked in effect as a way for students to conduct focused tests of their mechanisms [8]. They also provided a chance for educators, either in person or through design of digital simulation, to put a thumb on the scales of iteration and focus testing on parameters or underlying structures that a novice might not attend to.

\section{Supporting small bets}

While understanding that artefacts are comprised of interacting but somewhat independent systems is a foundational idea of engineering practice, it is not evident to many novices. Seeing that the whole is comprised of parts is a basic step to focused testing. In the workshop, we saw that participant progression toward this realization could be supported through differing materials (laser-cut gears for the mechanism, paper for graphic elements, cardboard for structure) and activity structures (like tables set-up just for testing of motors) that decoupled subsystems or subcomponents of the computational papercraft projects. We saw that tools like the customized microcontroller, or LocoTap, allowed for quick plug-and-go testing, while still retaining a connection to underlying computation, and encouraged participants to engage something like felt like productive play. We also saw that the flexible and inexpensive nature of cardboard helped reduce perceived risks of messing up, allowing participants to consider and test multiple ideas.

These elements of computational papercrafting overlapped and together allowed participants to make conjectures and take risks in small iterative cycles around targeted aspects of the project to make what we are calling "small bets" they could then test. We saw that rapid prototyping and iteration was effective when used to build familiarity with tools or materials [28], like the LocoTap and cardboard gears. When focused around a single subsystem, it also allowed knowledge to build up that could transcend the project itself. We saw evidence of this in conversations like those reported above that developed conceptual models of how gears work, and in comments between participants, such as "it [the mechanism] turns, but it's not very smooth, [but] you know how it's supposed to work now." Angelica's comment that she learned 
the importance of measurement also demonstrates how valued practices of testing and refinement can be built through experience instead of instruction. These iterations were made productive particularly through facilitator moves like recommending that participants check a component before attaching to a project, or attaching a servo horn that helps a participant see the connection between an isolated component and their ultimate vision.

Importantly, "small bets" allowed work to move forward even when not all areas of the project were well understood, something that is common to professional engineering [7] and especially prevalent with novices. We believe this reduced stress and frustration by keeping difficult steps to avoid becoming choke points for building and learning. Small bets also allowed for participants to have greater autonomy and responsibility when testing and prototyping. Following the example of facilitators, by the end of workshop, participants would ask each other to bring more parts and seemed unafraid of negative results when testing their projects. Removing fear of mistakes by emphasizing the potential to learn and redo is important to persistence and learning in making [20]. Our analysis here shows that this is not accomplished only through facilitation and culture, but also through the materials and set-up at the core of a project.

\section{Implications}

As work in the computational papercraft workshop showed, technical supports for testing and refinement can be successful in supporting valued testing and refinement practices among novice designers. In summary, (a) use of the simulator and customized microcontroller allowed for consideration of multiple alternatives and for "trial-before-error", and (b) learners were able to conduct focused tests on subsystems of their paper machines, and to make "small bets," allowing them to keep initial ideas and designs fluid.

To facilitate "trial-before-error," we recommend that, when working with novice designers or those who have little experience with tools being introduced, educators provide resources for visualizing design possibilities in advance of building and testing. While sketching and mockups can help with this, more tailored resources can distribute expert knowledge about a domain, allowing novices to design more ambitiously, focus their design iterations, and recognize opportunities for learning. Here, a digital simulation of several types of mechanical movement, alongside mentor questions and gestures, helped STEAM camp participants visualize implications of design choices and understand not only potential problems, but ways in which papercraft projects could best fit both aesthetic and learning goals. These opportunities for trialbefore-error can be especially powerful for opening an expansive and productive design space, even when time is constrained or with novices whose technical knowledge and skill can limit the generativity of ideation and rough prototyping.

To support "small bets," we recommend that educators provide resources - like separate testing stations or tools customized for particular aspects of a project - that encourage novice designers to notice and test separate parts of systems. This can help novices understand that subsystems can be refined independent of the whole project. This also allows participants to dig more deeply into aspects that interest them and to continue making progress (in their projects and learning goals) even when faced with sticking points in some areas. At the STEAM camp, the use of lowcost materials and a customized microcontroller gave participants a chance to explore and take 
risks in ways that were important not just to building intuitions about new materials and figuring out the technical details of mechatronic creations, but also to refining aesthetics and taking up an ethos of envisioning, trying and testing. We believe these are important to crafting not just objects, but identities and learning trajectories in STEAM.

This is not to say that participants completely or uniformly took up valued practices of iteration. For example, we saw few explicitly documented and/or measurable predictions $[29,36]$, and little reflection on strategies being used and lessons learned [8]. Supporting practices like these or identifying and labelling them when they arise [22] - will be an imperative in future workshop designs.

Nonetheless, the study sheds light on novice practices of testing and refinement, and how to support young people as they pursue personal learning goals within design education and move toward more sophisticated engineering and design practices. We believe it is important to continue identifying processes such as these in order to understand what iteration can and should be like in project-based, equity-oriented engineering education and design settings. Future research is needed to understand how and when these practices persist across activities, and how to support explicit reflection on iterative practices and their connection to interests, expertise and work in more formal STEM settings. There is also more work to be done to look across existing research to continue identifying pedagogical practices and outcomes relevant to learning-focused iteration. We also need to identify where effective practices of product-focused iteration might detract from youth learning in both short and long-term. In addition to the theoretical value of refining what processes of iteration might be most productive to support, we hope that the educator moves, activity structures and designs that appear in the moments described above will be useful to engineering educators.

\section{Acknowledgements}

This material is based upon work support by the National Science Foundation under Grant. No. 1735836 and the Gordon and Betty Moore Foundation. Special thanks to educator collaborators Carol Tang, Heather Madison, and Leah Henry; design collaborators HyunJoo Oh, Mike Eisenberg, and Ann Eisenberg; and interns Reneir Viray and Sarah Forman.

\section{References}

[1] D. C. Wynn and C. M. Eckert, "Perspectives on iteration in design and development," Res. Eng. Des., vol. 28, no. 2, pp. 153-184, 2017.

[2] C. Schimpf and C. Xie, "Characterizing Students' Micro-Iterations Strategies through Data-Logged Design Actions," in American Society for Engineering Education Annual Conference, 2017.

[3] R. Adams and C. Atman, "Characterizing Engineering Student Design Processes: An Illustration of Iteration," in American Society for Engineering Education Annual Conference, 2000.

[4] Y. Jin and P. Chusilp, "Study of mental iteration in different design situations," Des. Stud., vol. 27, no. 1, pp. 25-55, 2006.

[5] D. C. Wynn, C. M. Eckert, and P. J. Clarkson, "Modelling iteration in engineering design," in International Conference on Engineering Design (ICED), 2007. 
[6] S. Piccolo, S. L. Jørgensen, and A. Maier, "Using data and network science to reveal iterations and phase-transitions in the design process," in International Conference on Engineering Design (ICED), 2018.

[7] C. M. Cunningham and G. Y. J. Kelly, "Epistemic Practices of Engineering for Education," Sci. Educ., vol. 101, no. 3, 2017.

[8] D. P. Crismond and R. S. Adams, "The informed design teaching and learning matrix," $J$. Eng. Educ., vol. 101, no. 4, pp. 738-7979, 2012.

[9] N. R. Council, A Framework for K-12 Science Education: Practices, Crosscutting Concepts, and Core Ideas. Washington, DC: The National Academies Press, 2012.

[10] D. Crismond, "The Role of Diagnostic Reasoning in Engineering Design: A Case Study," in American Society for Engineering Education Annual Conference, 2008.

[11] R. P. Smith and P. Tjandra, "Experimental Observation of Iteration in Engineering Design,” Res. Eng. Des. - Theory, Appl. Concurr. Eng., vol. 10, pp. 107-117, 1998.

[12] R. Razzouk and V. Shute, "What is Design Thinking and Why Is It Important?," Rev. Educ. Res., vol. 82, no. 3, pp. 330-348, 2012.

[13] M. G. Guvench, "Computer Aided Design of Internally Compensated CMOS Operational Amplifiers," in American Society for Engineering Education Annual Conference, 2002.

[14] R. Adams, "Cognitive Processes in Iterative Design Behavior," University of Washington, Seattle, WA, 2001.

[15] C. J. Atman, M. E. Cardella, J. Turns, and R. Adams, "Comparing freshman and senior engineering design processes: an in-depth follow-up study," Des. Stud., vol. 26, no. 4, pp. 325-357, 2005.

[16] C. J. Atman, J. R. Chimka, M. Bursic, K., and L. (1999). Nachtmann, H., “A comparison of freshman and senior engineering design processes,” Des. Stud., vol. 20, pp. 131-152, 1999.

[17] T. R. Browning, "Process integration using the design structure matrix," Syst. Eng., vol. 5, no. 3, pp. 180-193, 2002.

[18] T. Brown, “Design Thinking," Harv. Bus. Rev., pp. 85-92, 2008.

[19] K. Wilkinson, L. Anzivino, and M. Petrich, "The Big Idea Is Their Idea," in Makeology: Makers as Learners, K. Peppler, E. R. Halverson, and Y. B. Kafai, Eds. New York: Routledge, 2016.

[20] J. J. Ryoo, N. Bulalacao, L. Kekelis, E. McLeod, and B. Henriquez, "Tinkering with 'Failure': Equity, Learning, and the Iterative Design Process,' FabLearn. Stanford, CA, 2015.

[21] A. Calabrese Barton, E. Tan, and D. Greenberg, "The Makerspace Movement: Sites of Possibilities for Equitable Opportunities to Engage Underrepresented Youth in STEM," Teach. Coll. Rec., vol. 119, no. 6, pp. 1-44, 2017.

[22] S. Vossoughi, M. Escude, F. Kong, and P. Hooper, "Tinkering, Learning \& Equity in the After-School Setting," FabLearn. Stanford University, 2013.

[23] C. Hutchinson, "Makerspaces: An Important Component of 21st Century Education," Haskell Insights, 2018. [Online]. Available: https://www.haskelloffice.com/makerspacesan-important-component-of-21st-century-education/. [Accessed: 12-Jan-2018].

[24] C. Costello, "STEM \& MakerSpace," Virtual Library, 2019.

[25] L. K. Berland and W. F. McKenna, "Student Responses to Challenge-based Engineering Curricula, Presented at Problem-based and Challenge-based Learning," in American Society for Engineering Education Annual Conference, 2012. 
[26] S. H. Kim and H. T. Zimmerman, "Collaborative Argumentation During a Making and Tinkering Afterschool Program with Squishy Circuits." 2017.

[27] K. Sheridan, E. Halverson, B. K. Litts, L. Brahms, L. Jacobs-Priebe, and T. Owens, "Learning in the Making: A Comparative Case Study of Three Makerspaces," Harv. Educ. Rev., vol. 84, no. 4, 2014.

[28] S. H. Kim and H. T. Zimmerman, "Towards a Stronger Conceptualization of the Maker Mindset: A case study of an afterschool program with squishy circuits," FabLearn. Stanford, CA, 2017.

[29] J. L. Kolodner et al., "Problem-Based Learning Meets Case-Based Reasoning in the Middle-School Science Classroom: Putting Learning by Design(tm) Into Practice," $J$. Learn. Sci., vol. 12, no. 4, pp. 495-547, 2003.

[30] S. P. Dow, K. Heddleston, and S. R. Klemmer, "The efficacy of prototyping under time constraints," in Proceeding of the seventh ACM conference on Creativity and cognition C\&C '09, 2009, p. 165.

[31] C. L. Dym, A. M. Agogino, O. Eris, D. D. Frey, and L. J. Leifer, "Engineering Design Thinking, Teaching, and Learning," J. Eng. Educ., vol. 94, no. 1, pp. 103-120, 2005.

[32] M. Eisenberg, H. Oh, S. Hsi, and M. Gross, "Paper mechatronics: A material and intellectual shift in educational technology," in 2015 International Conference on Interactive Collaborative Learning (ICL), 2015, pp. 936-943.

[33] H. Oh, M. D. Gross, and M. Eisenberg, "FoldMecha: Design for Linkage-Based Paper Toys," in Proceedings of the 28th Annual ACM Symposium on User Interface Software \& Technology - UIST'15 Adjunct, 2015, pp. 91-92.

[34] V. Braun and V. Clarke, "Using thematic analysis in psychology," Qual. Res. Psychol., vol. 3, no. 2, pp. 77-101, 2006.

[35] Merriam, S. (2009). Qualitative research: A guide to design and implementation (3 ed.). San Francisco: Jossey-Bass, pp. 178-186, 2009.

[36] C. Vieira, M. Hathaway Goldstein, Ş. Purzer, and A. J. Magana, "Using Learning Analytics to Characterize Student Experimentation Strategies in Engineering Design," J. Learn. Anal., vol. 3, no. 3, pp. 291-317, 2016.

[37] J. Corbin and A. Strauss, Basics of Qualitative Research: Second Edition: Techniques and Procedures for Developing Grounded Theory, 2nd ed. Thousand Oaks, CA: Sage Publications Inc., 1998.

[38] R. Stevens, K. O’Connor, L. Garrison, A. Jocuns, A., and D. Amos, "Becoming and Engineer: Toward a Three Dimensional View of Engineering Learning," J. Eng. Educ., July, pp. 363, 2008.

[39] D. Keifert and R. Stevens, "Inquiry as a Members' Phenomenon: Young Children as Competent Inquirers," J. Learn. Sci., 2018. 